SWAT-99/224

\title{
Group Approach to Quantization of Yang-Mills Theories: A Cohomological Origin of Mass *
}

\author{
Manuel Calixto ${ }^{1,3 \dagger}$ and Victor Aldaya ${ }^{2,3 \ddagger}$
}

1. Department of Physics, University of Wales Swansea, Singleton Park, Swansea, SA2 8PP, U.K.

2. Instituto de Astrofísica de Andalucía, Apartado Postal 3004, 18080 Granada, Spain.

3. Instituto Carlos I de Física Teórica y Computacional, Facultad de Ciencias, Universidad de Granada, Campus de Fuentenueva, 18002 Granada, Spain.

\begin{abstract}
New clues for the best understanding of the nature of the symmetry-breaking mechanism are revealed in this paper. A revision of the standard gauge transformation properties of Yang-Mills fields, according to a group approach to quantization scheme, enables the gauge group coordinates to acquire dynamical content outside the null mass shell. The corresponding extra (internal) field degrees of freedom are transferred to the vector potentials to conform massive vector bosons.
\end{abstract}

PACS: 11.15.-q, 03.65.Fd, 02.20.Tw, 11.15.Ex

KEYWORDS: gauge theories, symmetry breaking, groups, cohomology, algebraic quantization.

\section{Introduction}

Despite the undoubted success of the Standard Model in describing strong and electroweak interactions, a real (versus artificial) mechanism of mass-generation is still lacking. Needless to say that the discovery of a Higgs boson - a quantum vibration of an abnormal (Higgs) vacuum - would be of enormous importance; nevertheless, at present, no dynamical basis for the Higgs mechanism exists, and it is purely phenomenological. It is true that there is actually nothing inherently unreasonable in the idea that the state of

\footnotetext{
${ }^{*}$ Work partially supported by the DGICYT.

†E-mail: pymc@swansea.ac.uk / calixto@ugr.es

${ }^{\ddagger}$ E-mail: valdaya@iaa.es
} 
minimum energy $|\tilde{0}\rangle$ (the vacuum) may be one in which some field quantity $\hat{\varphi}(x)$ has a non-zero expectation value $\langle\tilde{0}|\hat{\varphi}(x)| \tilde{0}\rangle=\varphi_{0}$; in fact, many examples in condensed-matter physics display this feature. Nevertheless, it remains conjectural whether something similar actually happens in the weak interaction case.

Also, the ad hoc introduction of extra (Higgs) scalar fields in the theory to provide mass to the vector bosons could be seen as our modern equivalent of those earlier mechanical contrivances populating the plenum (the ether), albeit very subtly. As in those days, new perspectives are necessary to explain why it is really not indispensable to look at things this way at all.

This paper provides a new approach to quantum Yang-Mills theories, from a grouptheoretic perspective, in which mass enters the theory in a natural way; more precisely, the presence of mass will manifest through non-trivial transformations of the phase $\zeta=$ $e^{i \alpha}$ of the wave functional $\Psi(A)$ under the action of gauge transformations. This nontrivial response of the phase under gauge transformations causes a deformation of the corresponding Lie-algebra commutators and leads to the appearance of central terms proportional to mass parameters and, consequently, to a quantum generation of extra (internal) field degrees of freedom according to a self-interacting theory of massless and massive vector bosons (without Higgs fields).

This cohomological mechanism of mass-generation makes perfect sense from a Group Approach to Quantization (GAQ [1]) framework, and we shall use its concepts and tools to work out the quantization of Yang-Mills theories. Given that this is not a common approach to quantization, we shall give useful references and try to be as self-contained as possible (the reader is advised to have a look at the Ref. [2], which contains a general presentation of GAQ for linear fields). Quantizing on a group, however, will require the revision of some standard concepts, such as gauge transformations, in order to deal with them properly. The meaning of gauge transformations in Quantum Mechanics is not well understood at present (see, for example, [3]); thus, a re-examination of it is timely.

Gauge symmetry is always a guarantee for the renormalizability of a field theory. The introduction of mass usually spoils gauge invariance, but the Higgs mechanism manages to preserve renormalizability by keeping gauge invariance in a hidden way, and this is the main novelty in comparison with other attempts to supply mass. However, we must say that the breakdown of a gauge symmetry and the appearance of anomalous (unexpected) situations are sometimes subtle questions which generally go with the standard approach of quantizing classical systems. From a group-theoretic framework, any consistent (nonperturbative) quantization is just a unitary irreducible representation of a suitable (Lie, Poisson) algebra. This approach does not assume the existence of a previous classical underlying system and overcomes some of the standard failures in quantization (anomalies) attached to canonical quantization, reinterpreting them as normal (even essential) situations.

A unified quantization of massless and massive non-Abelian vector bosons will be presented in Sections 3 and 4, respectively; the Abelian case (Electromagnetic and Proca fields) is briefly discussed in Sec. 2. The Hilbert space of the theory is related to the carrier space of the unitary irreducible representations of an infinite-dimensional quantizing group 
$\tilde{G}$, the mass eventually being a parameter characterizing the representation. Section 5 is devoted to the incorporation of fermionic matter into the theory. Finally, we conclude in Section 6 and incorporate an Appendix with a simple, but illustrative, finite-dimensional analogy.

\section{The Abelian case}

In a previous article [4], a revision of the traditional concept of gauge transformation for the electromagnetic vector potential,

$$
\varphi(x) \rightarrow \varphi^{\prime}(x)+\varphi(x), \quad A_{\mu}(x) \rightarrow A_{\mu}(x)-\partial_{\mu} \varphi^{\prime}(x),
$$

was necessary to arrange this transformation inside a group law that is, to adapt this operation to an action of a group on itself: the group law of the (infinite-dimensional) electromagnetic quantizing group $\tilde{G}$. The proposed Lie group $\tilde{G}$ had a principal bundle structure $\tilde{G} \rightarrow \tilde{G} / \tilde{T}$ and was parameterized, roughly speaking, by the coordinates $A_{\mu}(\vec{x}, t)$ of the Abelian subgroup $G_{A}$ of Lie algebra valued vector potentials, the coordinates $v=$ $\left(y_{\mu}, \Lambda_{\mu \nu}\right)$ (space-time translations and Lorentz transformations) of the Poincaré group $P$ and the coordinates $\varphi(\vec{x}, t)$ of the local group $T \equiv U(1)(\vec{x}, t)$, which took part of the structure group $\tilde{T} \sim T \times U(1)$ and generalized the standard $U(1)$-phase invariance, $\Psi \sim e^{i \alpha} \Psi$, in Quantum Mechanics. In this way, the extra $\tilde{T}$-equivariance conditions on wave functions [complex valued functions $\Psi(\tilde{g})$ on $\tilde{G}]$, i.e. $\Psi\left(\tilde{g}_{t} * \tilde{g}\right) \sim \Psi(\tilde{g}), \tilde{g}_{t} \in \tilde{T}$, provided the traditional constraints of the theory.

The abovementioned revision was motivated by the fact that the transformation (1) is not compatible with a group law. Indeed, the general property $g * e=e * g=g$ for a composition law $g^{\prime \prime}=g^{\prime} * g$ of a group $G$ ( $e$ denotes the identity element), precludes the existence of linear terms, in the group law $g^{\prime \prime j}=g^{\prime \prime j}\left(g^{\prime k}, g^{l}\right)$ of a given parameter $g^{j}$ of $G$, other than $g^{\prime j}$ and $g^{j}$; that is, near the identity we have $g^{\prime \prime j}=g^{\prime j}+g^{j}+O(2)$. Therefore, the group law for the field parameter $A_{\mu}$ cannot have linear terms in $\varphi$. The natural way to address this situation is to leave the vector potential unchanged, and change the phase $\zeta=e^{i \alpha}$ of the quantum-mechanical wave functional $\Psi(A)$ as follows:

$$
\begin{array}{r}
\varphi(x) \rightarrow \varphi(x)+\varphi^{\prime}(x), \quad A_{\mu}(x) \rightarrow A_{\mu}(x), \\
\zeta \rightarrow \zeta \exp \left\{-\frac{i}{2 c \hbar^{2}} \int_{\Sigma} d \sigma_{\mu}(x) \eta^{\rho \sigma} \partial_{\rho} \varphi^{\prime}(x) \overleftrightarrow{\partial^{\mu}} A_{\sigma}(x)\right\},
\end{array}
$$

where $\eta^{\rho \sigma}$ denotes the Minkowski metric, $\Sigma$ denotes a spatial hypersurface and $\hbar$ is the Planck constant, which is required to kill the dimensions of $\partial_{\rho} \varphi^{\prime} \overleftrightarrow{\partial^{\mu}} A^{\rho} \equiv \partial_{\rho} \varphi^{\prime} \partial_{\mu} A^{\rho}-$ $A^{\rho} \partial_{\mu} \partial_{\rho} \varphi^{\prime}$ and gives a quantum character to the transformation (2) versus the classical character of (1) [hereafter, we shall use natural unities $\hbar=1=c$ ]. The piece $\partial_{\rho} \varphi^{\prime} \overleftrightarrow{\partial^{\mu}} A^{\rho}$ takes part of a symplectic current

$$
J^{\mu}\left(g^{\prime} \mid g\right)(x) \equiv \frac{1}{2} \eta^{\rho \sigma}\left[\left(v A^{\prime}\right)_{\rho}(x)-\partial_{\rho}\left(v \varphi^{\prime}\right)(x)\right] \overleftrightarrow{\partial^{\mu}}\left[A_{\sigma}(x)-\partial_{\sigma} \varphi(x)\right],
$$


[we are denoting $g \equiv(A, \varphi, v)$ and $\left(v A^{\prime}\right)_{\rho}(x) \equiv \frac{\partial v^{\alpha}(x)}{\partial x^{\rho}} A_{\alpha}^{\prime}(v(x)),\left(v \varphi^{\prime}\right)(x) \equiv \varphi^{\prime}(v(x))$, with $v^{\alpha}(x)=\Lambda_{\beta}^{\alpha} x^{\beta}+y^{\alpha}$ the general action of the restricted Poincaré group $P$ on Minkowski space-time] which is conserved, $\partial_{\mu} J^{\mu}=0$, if $A_{\nu}$ and $\varphi$ satisfy the field equations $\left(\partial_{\mu} \partial^{\mu}+\right.$ $\left.m^{2}\right) A_{\nu}=0$ and $\left(\partial_{\mu} \partial^{\mu}+m^{2}\right) \varphi=0$ ( $m$ is a parameter with mass dimension), so that the integral in (2) does not depend on the chosen space-like hypersurface $\Sigma$. The integral $\xi\left(g^{\prime} \mid g\right) \equiv \int_{\Sigma} d \sigma_{\mu}(x) J^{\mu}\left(g^{\prime} \mid g\right)(x)$ is a two-cocycle $\xi: G \times G \rightarrow \Re[G$ denotes the semi-direct product $\left.\left(G_{A} \times T\right) \times{ }_{v} P\right]$, which fulfis the well-known properties:

$$
\begin{gathered}
\xi\left(g^{\prime} \mid g\right)+\xi\left(g^{\prime} * g \mid g^{\prime \prime}\right)=\xi\left(g^{\prime} \mid g * g^{\prime \prime}\right)+\xi\left(g \mid g^{\prime \prime}\right), \quad \forall g, g^{\prime}, g^{\prime \prime} \in G, \\
\xi(g \mid e)=0=\xi(e \mid g), \quad \forall g \in G,
\end{gathered}
$$

and is the basic ingredient to construct the centrally extended group law $\tilde{g}^{\prime \prime}=\tilde{g}^{\prime} * \tilde{g}$, more explicitly

$$
\tilde{g}^{\prime \prime} \equiv\left(g^{\prime \prime} ; \zeta^{\prime \prime}\right)=\left(g^{\prime} * g ; \zeta^{\prime} \zeta e^{i \xi\left(g^{\prime} \mid g\right)}\right), \quad g, g^{\prime}, g^{\prime \prime} \in G ; \zeta, \zeta^{\prime}, \zeta^{\prime \prime} \in U(1),
$$

of the electromagnetic quantizing group $\tilde{G}$ (see below and Ref. [4] for more details).

It bears mentioning that the required revision of the concepts of gauge transformations and constraint conditions to construct the quantizing group $\tilde{G}$ has led, as a byproduct, to a unified quantization of both the electromagnetic and Proca fields [4], within the same general scheme of quantization based on a group (GAQ) [1]. The different structure of the central extension (5) for the massive case, with regard the massless case, manifests itself through a true (non-trivial) central-extension $\tilde{T}$ of the constraint subgroup $T$ by $U(1)$ given by the peace

$$
\xi_{m}\left(g^{\prime} \mid g\right)=\frac{1}{2} \int_{\Sigma} d \sigma_{\mu} \eta^{\rho \sigma} \partial_{\rho}\left(v \varphi^{\prime}\right) \overleftrightarrow{\partial^{\mu}} \partial_{\sigma} \varphi=\frac{m^{2}}{2} \int_{\Sigma} d \sigma_{\mu}\left(v \varphi^{\prime}\right) \overleftrightarrow{\partial^{\mu}} \varphi
$$

of the cocycle $\xi\left(g^{\prime} \mid g\right) \equiv \int_{\Sigma} d \sigma_{\mu} J^{\mu}\left(g^{\prime} \mid g\right)$. The piece $\xi_{m}$, which is one $\left(\xi_{3}\right)$ of the three typical and distinguishable pieces $\left(\xi_{j}, j=1,2,3\right)$ in which $\xi$ splits up (see [4,5] and bellow), gives dynamics to the local group $T$ (creates new couples of conjugated variables), and makes the constraints of second-class nature. This results in an increased number of field degrees of freedom with regard the massless case, leading to a Proca quantum field (see [4] for more details).

Furthermore, the standard (classical) transformation (1) is regained as the trajectories associated with generalized equations of motion generated by vector fields with null Noether invariants (gauge subalgebra, see Refs. [4, 2] and [5] for a formal exposition, including tensor fields).

A unified scheme of quantization for non-Abelian massless and massive vector bosons is also possible in this scheme and suitable as an alternative to the standard Spontaneous Symmetry Breaking mechanism, which is intended to supply mass while preserving renormalizability. However, for this case, the situation seems to be a bit more subtle and far richer. 


\section{Group-quantization of Yang-Mills Fields}

According to the headings in the foregoing section, our main purpose now is to offer a reasonable attempt to find a (non-perturbative) unified quantization of non-Abelian massless and massive vector bosons without Higgs fields. As in the Abelian case, the key to achieve this goal consists in a revision of the traditional concept of gauge transformation for vector potentials,

$$
U(x) \rightarrow U^{\prime}(x) U(x), \quad A_{\nu}(x) \rightarrow U^{\prime}(x) A_{\nu}(x) U^{\prime}(x)^{-1}+U^{\prime}(x) \partial_{\nu} U^{\prime}(x)^{-1},
$$

in order to make it compatible with a group law: the group law of the (infinite-dimensional) Yang-Mills quantizing group $\tilde{G}$, which will be the primary object to define the quantum theory. This group has a fibre-bundle structure $\tilde{G} \rightarrow \tilde{G} / \tilde{T}$ and is parametrized, roughly speaking, by the coordinates $A^{\mu}(x)=r_{a}^{b} A_{b}^{\mu}(x) T^{a}$ of an Abelian subgroup $G_{A}$ of Lie algebra valued vector potentials $\left[r_{a}^{b}\right.$ is a coupling-constant matrix and $T^{a}$ are the Lie-algebra generators of the rigid subgroup $\mathbf{T}$, of a gauge group $T$, satisfying the commutation relations $\left[T^{a}, T^{b}\right]=C_{c}^{a b} T^{c}$ and defining the structure constants $\left.C_{c}^{a b}\right]$ and the coordinates $U(x)=e^{\varphi_{a}(x) T^{a}} \equiv e^{\varphi(x)}$ of the local group $T$, which takes part of the structure subgroup $\tilde{T} \sim T \times U(1)$ and generalizes the standard $U(1)$-phase invariance $\Psi \sim e^{i \alpha} \Psi$ in Quantum Mechanics as a particular case of $\tilde{T}$-equivariance condition [6]

$$
\Psi\left(\tilde{g}_{t} * \tilde{g}\right)=D_{\tilde{T}}^{(\epsilon)}\left(\tilde{g}_{t}\right) \Psi(\tilde{g}), \quad \forall \tilde{g}_{t} \in \tilde{T}, \forall \tilde{g} \in \tilde{G}
$$

on complex wave functionals $\Psi: \tilde{G} \rightarrow C$ defined on $\tilde{G}$, where $D_{\tilde{T}}^{(\epsilon)}$ symbolizes a specific representation $D$ of $\tilde{T}$ with $\epsilon$-index (in particular, the $\epsilon=\vartheta$-angle [7] of non-Abelian gauge theories; see below). As already commented, the $\tilde{T}$-equivariance conditions (8) provide the traditional constraints of the theory, which will be first- or second-class depending on whether the fribration of the structure subgroup $\tilde{T} \rightarrow \tilde{T} / U(1)$ by $U(1)$ is trivial or not ( $m=0$ or $m \neq 0$, respectively; see below).

As mentioned above in the Abelian case, the transformation (7) is not compatible with a group law. The natural way to adapt the operation (7) to an action of a group on itself is to consider that $A_{\nu}$ transforms homogeneously under the adjoint action of $T$, whereas the non-tensorial part $U(x) \partial_{\nu} U^{\prime}(x)^{-1}$ modifies the phase $\zeta=e^{i \alpha}$ of the wave functional $\Psi(A)$ according to:

$$
\begin{gathered}
U(x) \rightarrow U^{\prime}(x) U(x), \quad A_{\nu}(x) \rightarrow U^{\prime}(x) A_{\nu}(x) U^{\prime}(x)^{-1} \\
\zeta \rightarrow \zeta \exp \left\{\frac{i}{r^{2}} \int_{\Sigma} d \sigma_{\mu}(x) \operatorname{tr}\left[U^{\prime}(x)^{-1} \partial_{\nu} U^{\prime}(x) \overleftrightarrow{\partial^{\mu}} A^{\nu}(x)\right]\right\}
\end{gathered}
$$

We are restricting ourselves, for the sake of simplicity, to gauge groups $T$ associated with rigid special unitary groups $\mathbf{T}$ for which the structure constants $C_{c}^{a b}$ are totally antisymmetric, and the anti-hermitian generators $T^{a}$ can be chosen such that the KillingCartan metric is just $\operatorname{tr}\left(T^{a} T^{b}\right)=-\frac{1}{2} \delta^{a b}$. For simple groups, the coupling-constant matrix

$r_{a}^{b}$ reduces to a multiple of the identity $r_{a}^{b}=r \delta_{a}^{b}$, and we have $A_{a}^{\mu}=-\frac{2}{r} \operatorname{tr}\left(T^{a} A^{\mu}\right)$. The 
argument of the exponential in (9) can be considered to be a piece of a two-cocycle $\xi: G \times G \rightarrow \Re\left(G\right.$ is the semi-direct product $G=G_{A} \times_{U} T$ of $T$ and the Abelian group $G_{A}$ of Lie-algebra valued potentials) constructed through a conserved current, $\xi\left(g^{\prime} \mid g\right)=\int_{\Sigma} d \sigma_{\mu}(x) J^{\mu}\left(g^{\prime} \mid g\right)(x), g^{\prime}, g \in G$, so that it does not depend on the chosen spacelike hypersurface $\Sigma$ (see $[2,5])$. On this basis, let us construct a central extension $\tilde{G}$ of $G$ by making use of a two-cocycle defined on the particular $t=$ constant $\Sigma$-hypersurface. We shall also make partial use of the gauge freedom to set the temporal component $A^{0}=0$, so that the electric field is simply $\vec{E}_{a}=-\partial_{0} \vec{A}_{a}$ [from now on, and for the sake of simplicity, we shall put any three-vector $\vec{A}$ as $A$, and understand $A E=\sum_{j=1}^{3} A^{j} E^{j}$, in the hope that no confusion will arise]. In this case, there is still a residual gauge invariance $T=\operatorname{Map}\left(\Re^{3}, \mathbf{T}\right)$ (see [8]).

The explicit group law $\tilde{g}^{\prime \prime}=\tilde{g}^{\prime} * \tilde{g}$ [with $\left.\tilde{g}=(g ; \zeta)=(A, E, U ; \zeta)\right]$ for the proposed infinite-dimensional Yang-Mills quantizing group $\tilde{G}$ is:

$$
\begin{aligned}
& U^{\prime \prime}(x)=U^{\prime}(x) U(x), \\
& A^{\prime \prime}(x)=A^{\prime}(x)+U^{\prime}(x) A(x) U^{\prime}(x)^{-1}, \\
& E^{\prime \prime}(x)=E^{\prime}(x)+U^{\prime}(x) E(x) U^{\prime}(x)^{-1} \text {, } \\
& \zeta^{\prime \prime}=\zeta^{\prime} \zeta \exp \left\{-\frac{i}{r^{2}} \sum_{j=1}^{3} \xi_{j}\left(A^{\prime}, E^{\prime}, U^{\prime} \mid A, E, U\right)\right\} ; \\
& \xi_{1}\left(g^{\prime} \mid g\right) \equiv \int d^{3} x \operatorname{tr}\left[\left(\begin{array}{cc}
A^{\prime} & E^{\prime}
\end{array}\right) S\left(\begin{array}{c}
U^{\prime} A U^{\prime-1} \\
U^{\prime} E U^{\prime-1}
\end{array}\right)\right], \\
& \xi_{2}\left(g^{\prime} \mid g\right) \equiv \int d^{3} x \operatorname{tr}\left[\left(\begin{array}{cc}
\nabla U^{\prime} U^{\prime-1} & E^{\prime}
\end{array}\right) S\left(\begin{array}{c}
U^{\prime} \nabla U U^{-1} U^{\prime-1} \\
U^{\prime} E U^{\prime-1}
\end{array}\right)\right], \\
& \xi_{3}\left(g^{\prime} \mid g\right) \equiv-2 \int d^{3} x \operatorname{tr}\left[\lambda\left(\log \left(U^{\prime} U\right)-\log U^{\prime}-\log U\right)\right],
\end{aligned}
$$

where $S=\left(\begin{array}{cc}0 & 1 \\ -1 & 0\end{array}\right)$ is a symplectic matrix and $\lambda \equiv \lambda_{a} T^{a}$ is a linear function (a matrix) on the Cartan subalgebra of the rigid subgroup $\mathbf{T}$ of $T$.

We have split up the two-cocycle $\xi$ into three significantly distinguishable two-cocycles $\xi_{j}, j=1,2,3$ (as in $[4,5]$ ) for a much better understanding. The first two-cocycle $\xi_{1}$ is meant to provide dynamics to the vector potential, so that the couple $(A, E)$ corresponds to a canonically-conjugate pair of coordinates. The second two-cocycle $\xi_{2}$, the mixed two-cocycle, provides a non-trivial (non-diagonal) action of the structure subgroup $T$ on vector potentials and determines the number of degrees of freedom of the constrained theory; it is the non-covariant analogue of the argument of the exponential in (9). Both cocycles correspond to the analogous ones of the Abelian case. Concerning the third one, $\xi_{3} \equiv \xi_{\lambda}$, its origin and nature departs essentially from the Abelian "analogue" (6). Unlike the Abelian case $T=U(1)(x)$, the semi-simple character of $\mathbf{T}$ precludes a true central extension $\tilde{T}$ of $T=\operatorname{Map}\left(\Re^{3}, \mathbf{T}\right)$ by $U(1)$ (this is not the case in one compact spatial dimension $\Re^{3} \leftrightarrow S^{1}$, where true central extensions are known for Kac-Moody groups). 
However, there exists certain coboundaries, called pseudo-cocycles, which define trivial extensions as such, but provide new commutation relations in the Lie algebra of $\tilde{G}$ and provide a non-trivial piece of the connection form of the theory [1],

$$
\Theta=\left.\frac{\partial}{\partial g^{j}} \xi\left(g^{\prime} \mid g\right)\right|_{g^{\prime}=g^{-1}} d g^{j}-i \zeta^{-1} d \zeta,
$$

thus altering, in particular, the number of degrees of freedom of the theory (see [9] for a relationship between pseudo-cohomology and coadjoint orbits of semisimple groups). This is precisely the case of the third cocycle (coboundary, indeed), $\xi_{3}\left(g^{\prime} \mid g\right)=\eta\left(g^{\prime} * g\right)-$ $\eta\left(g^{\prime}\right)-\eta(g)$, generated by a function $\eta(g)=-2 \int d^{3} x \operatorname{tr}[\lambda \log U]$ with non-trivial gradient $\left.\delta \eta(g)\right|_{g=e}=\left.\frac{\delta \eta(g)}{\delta g^{j}}\right|_{g=e} \delta g^{j} \neq 0$ at the identity $g=e$, which is locally linear in the parameters of the Cartan subgroup with as many independent coefficients (constants) $\lambda_{a}$ as elements in the Cartan subalgebra, i.e. the range of the rigid group $\mathbf{T}$. The introduction of such a pseudo-cocycle is needed to obtain a faithful representation of the rigid subgroup $\mathbf{T}$, according to our general group representation approach. Pseudo-cocycles similar to $\xi_{3}$ do appear in the representation of Kac-Moody groups and in conformally invariant theories in general, although the pseudo-cocycle parameters are usually hidden in a redefinition of the generators involved in the pseudo-extension (the argument of the Lie-algebra generating function). This is the case of the Virasoro algebra in String Theory,

$$
\left[\hat{L}_{n}, \hat{L}_{m}\right]=(n-m) \hat{L}_{n+m}+1 / 12\left(c n^{3}-c^{\prime} n\right) \delta_{n,-m} \hat{1},
$$

where the $\hat{L}_{0}$ generator is redefined so as to produce a non-trivial expectation value in the vacuum, $h \equiv\left(c-c^{\prime}\right) / 24[10]$.

The cocycle $\xi_{3}$, however, for $\lambda \neq 0$, again determines the structure of constraints (firstor second-class) and modifies the dynamical content of the vector potential coordinates $A$ by transferring degrees of freedom between the $A$ and $\varphi$ coordinates. As in the Abelian case, this mechanism conforms massive vector bosons so that $\xi_{3}$ must be considered as a mass cocycle. In this way, the appearance of mass in the theory has a cohomological origin. Notice that the parameter $\lambda\left(\lambda_{a}\right)$ bears the dimensions of cubed mass (in natural unities) and can well be renamed by $m^{3}\left(m_{a}^{3}\right)$.

To make more explicit the intrinsic significance of these three quantities $\xi_{j}, j=1,2,3$, let us calculate the non-trivial Lie-algebra commutators of the right-invariant vector fields (that is, the generators of the left-action $L_{\tilde{g}^{\prime}}(\tilde{g})=\tilde{g}^{\prime} * \tilde{g}$ of $\tilde{G}$ on itself) from the group law (10). They are explicitly:

$$
\begin{aligned}
{\left[\tilde{X}_{A_{a}^{j}(x)}^{R}, \tilde{X}_{E_{b}^{k}(y)}^{R}\right] } & =-\delta^{a b} \delta_{j k} \delta(x-y) \Xi \\
{\left[\tilde{X}_{E_{a}(x)}^{R}, \tilde{X}_{\varphi_{b}(y)}^{R}\right] } & =-C_{c}^{a b} \delta(x-y) \tilde{X}_{E_{c}(x)}^{R}+\frac{1}{r} \delta^{a b} \nabla_{x} \delta(x-y) \Xi \\
{\left[\tilde{X}_{A_{a}(x)}^{R}, \tilde{X}_{\varphi_{b}(y)}^{R}\right] } & =-C_{c}^{a b} \delta(x-y) \tilde{X}_{A_{c}(x)}^{R} \\
{\left[\tilde{X}_{\varphi_{a}(x)}^{R}, \tilde{X}_{\varphi_{b}(y)}^{R}\right] } & =-C_{c}^{a b} \delta(x-y) \tilde{X}_{\varphi_{c}(x)}^{R}-C_{c}^{a b} \frac{\lambda^{c}}{r^{2}} \delta(x-y) \Xi,
\end{aligned}
$$


where we denote by $\Xi \equiv i \tilde{X}_{\zeta}^{L}=i \tilde{X}_{\zeta}^{R}$ the central generator, in order to distinguish it from the rest, in view of its crucial role in the quantization procedure; it behaves as $i$ times the identity operator, $\Xi \Psi(\tilde{g})=i \Psi(\tilde{g})$, when the $U(1)$ part of the $\tilde{T}$-equivariance conditions $(8), D_{\tilde{T}}^{(\epsilon)}(\zeta)=\zeta$ (always faithful, except in the classical limit $U(1) \rightarrow \Re[1]$ ), is imposed. The commutators (13) agree with those of Ref. [8] when $\lambda_{c}=0$ and the identification $\hat{E}_{a} \equiv i \tilde{X}_{A_{a}}^{R}, \hat{A}_{a} \equiv i \tilde{X}_{E_{a}}^{R}, \hat{G}_{a} \equiv i \tilde{X}_{\varphi_{a}}^{R}$ is made [note that $\tilde{X}_{A_{a}}^{R} \sim \frac{\delta}{\delta A_{a}}$ and $\tilde{X}_{E_{a}}^{R} \sim \frac{\delta}{\delta E_{a}}$ near the identity element $\tilde{g}=e$ of $\tilde{G}$, which motivates this particular identification]. From the last line of (13) we realize that the pseudo-cocycle $\xi_{3}$ introduces new central terms proportional to the mass parameters $\lambda_{c}=m_{c}^{3}$, with respect to the massless case, which provide new "conjugated" coordinates; that is, extra degrees of freedom enter the theory through this pseudo-extension, which provides dynamics to the local (gauge) coordinates $\varphi_{a}$ of the structure subgroup $\tilde{T}$, dynamics which is transferred to the vector potentials $A_{a}$ to conform massive vector bosons.

To understand fully the last statement concerning the interplay among different cocycles and mainly between the massless and massive cases, we must construct the Hilbert space of both theories explicitly. Let us proceed with the massless case and leave the peculiarities of the massive one to the next section.

The representation $L_{\tilde{g}^{\prime}} \Psi(\tilde{g})=\Psi\left(\tilde{g}^{\prime} * \tilde{g}\right)$ of $\tilde{G}$ on $\tilde{T}$-equivariant wave functions (8) proves to be reducible. The reduction can be achieved by means of those right conditions ("polarization conditions" [1]) $R_{\tilde{g}_{p}} \Psi\left(\tilde{g}^{\prime}\right)=\Psi\left(\tilde{g}^{\prime} * \tilde{g}_{p}\right) \equiv \Psi\left(\tilde{g}^{\prime}\right) \forall \tilde{g}^{\prime} \in \tilde{G}$ compatible with the $\tilde{T}$-equivariant conditions (8), in particular with $\Xi \Psi=i \Psi$. In general, polarization conditions contain finite right-transformations generated by left-invariant vector fields $\tilde{X}^{L}$ devoid of dynamical content (that is, without a conjugated counterpart), and half of the left-invariant vector fields related to dynamical coordinates (either "positions" or "momenta"). The left-invariant vector fields without conjugated counterpart are the combinations

$$
\mathcal{G}_{c} \equiv<\tilde{X}_{\theta_{a}}^{L} \equiv \tilde{X}_{\varphi_{a}}^{L}-\frac{1}{r} \nabla \cdot \tilde{X}_{A_{a}}^{L}, / C_{c}^{a b} \lambda^{c}=0 \forall b>.
$$

The characteristic subalgebra $\mathcal{G}_{c}$ can be completed to a full polarization subalgebra $\mathcal{G}_{p}$ in two different ways:

$$
\mathcal{G}_{p}^{(A)} \equiv<\tilde{X}_{\theta_{a}}^{L} \in \mathcal{G}_{c}, \tilde{X}_{A_{b}}^{L} \forall b>, \quad \mathcal{G}_{p}^{(E)} \equiv<\tilde{X}_{\theta_{a}}^{L} \in \mathcal{G}_{c}, \tilde{X}_{E_{b}}^{L} \forall b>
$$

each one giving rise to a different representation space: a) the electric field representation and b) the magnetic field representation, respectively.

\section{a) The electric field representation $\Psi_{A}$.}

The solution to the polarization conditions $R_{\tilde{g}_{p}} \Psi_{A}(\tilde{g})=\Psi_{A}(\tilde{g}), \forall \tilde{g}_{p} \in G_{p}^{(A)}, \forall \tilde{g} \in \tilde{G}$ or, in infinitesimal form $\tilde{X}^{L} \Psi_{A}=0, \forall \tilde{X}^{L} \in \mathcal{G}_{p}^{(A)}$, proves to be:

$$
\Psi_{A}(A, E, U ; \zeta)=\zeta e^{-\frac{i}{r^{2}} \int d^{3} x \operatorname{tr}\left[A E-U \nabla U^{-1} E\right]} \Phi_{A}(E)
$$


where $\Phi_{A}$ is an arbitrary functional of $E$. The left-action of a general element $\tilde{g}^{\prime}=$ $\left(A^{\prime}, E^{\prime}, U^{\prime} ; \zeta^{\prime}\right)$ of $\tilde{G}$ on wave functions $\Psi_{A}$ is:

$$
\begin{aligned}
L_{\tilde{g}^{\prime}} \Psi_{A}(\tilde{g})= & \zeta^{\prime} \zeta e^{-2 \frac{i}{r^{2}} \int d^{3} x \operatorname{tr}\left[A^{\prime} U^{\prime} E U^{\prime-1}+U^{\prime-1} \nabla U^{\prime} E+\frac{1}{2} A^{\prime} E^{\prime}\right]} \\
& \cdot e^{-\frac{i}{r^{2}} \int d^{3} x \operatorname{tr}\left[A E-U \nabla U^{-1} E\right]} \Phi_{A}\left(E^{\prime}+U^{\prime} E U^{\prime-1}\right) .
\end{aligned}
$$

The particular case of $\tilde{g}^{\prime}=\tilde{g}_{t}{ }^{\prime}=\left(0,0, U^{\prime} ; 1\right) \in T \subset \tilde{T}$ gives us the expression of the rest of $\tilde{T}$-equivariant conditions (8), i.e. the constraint equations:

$$
L_{\tilde{g}_{t}^{\prime}} \Psi_{A}(\tilde{g})=D_{\tilde{T}}^{(\epsilon)}\left(\tilde{g}_{t}^{\prime}\right) \Psi_{A}(\tilde{g}) \Rightarrow \Phi_{A}(E)=e^{-2 \frac{i}{r^{2}} \int d^{3} x \operatorname{tr}\left[U^{\prime-1} \nabla U^{\prime} E\right]} \Phi_{A}\left(U^{\prime} E U^{\prime-1}\right),
$$

where we have chosen the trivial representation $D_{\tilde{T}}^{(\epsilon)}=1$ for $T$ (see below for more general cases).

The polarized, $\tilde{T}$-equivariant wave functions $(16,18)$ define the constrained Hilbert space $\mathcal{H}(\tilde{G})$ of the theory, and the infinitesimal form $\tilde{X}_{\tilde{g}^{\prime}}^{R} \Psi$ of the finite left-action $L_{\tilde{g}^{\prime}} \Psi(\tilde{g})$ of $\tilde{G}$ on $\mathcal{H}(\tilde{G})$ provides the action of the operators $\hat{A}_{a}, \hat{E}_{a}, \hat{G}_{a}$ on wave functions. Thus, the group $\tilde{G}$ is irreducibly and unitarily represented with respect to the natural scalar product $\left\langle\Psi \mid \Psi^{\prime}\right\rangle=\int_{\tilde{G}} \mu(\tilde{g}) \Psi^{*}(\tilde{g}) \Psi^{\prime}(\tilde{g})$, where $\mu(\tilde{g})$ denotes the standard left-invariant measure of $\tilde{G}$ [exterior product of the components of the left-invariant 1 -form $\theta^{L}$ ].

The infinitesimal form of the finite expressions (17) is:

$$
\begin{aligned}
\tilde{X}_{A_{a}}^{R} \Psi_{A} & =\zeta e^{-\frac{i}{r^{2}} \int d^{3} x \operatorname{tr}\left[A E-U \nabla U^{-1} E\right]} i E_{a} \Phi_{A}(E) \Rightarrow \hat{E}_{a} \Phi_{A}(E)=-E_{a} \Phi_{A}(E) \\
\tilde{X}_{E_{a}}^{R} \Psi_{A} & =\zeta e^{-\frac{i}{r^{2}} \int d^{3} x \operatorname{tr}\left[A E-U \nabla U^{-1} E\right]} \frac{\delta}{\delta E_{a}} \Phi_{A}(E) \Rightarrow \hat{A}_{a} \Phi_{A}(E)=i \frac{\delta}{\delta E_{a}} \Phi_{A}(E) \\
\tilde{X}_{\varphi_{a}}^{R} \Psi_{A} & =\zeta e^{-\frac{i}{r^{2}} \int d^{3} x \operatorname{tr}\left[A E-U \nabla U^{-1} E\right]}\left(-\frac{i}{r} \nabla \cdot E_{a}+C_{c}^{a b} E_{b} \cdot \frac{\delta}{\delta E_{c}}\right) \Phi_{A}(E) \\
& \Rightarrow \hat{G}_{a} \Phi_{A}(E)=\left(-\frac{1}{r} \nabla \cdot \hat{E}_{a}-C_{c}^{a b} \hat{E}_{b} \cdot \hat{A}_{c}\right) \Phi_{A}(E),
\end{aligned}
$$

which provides the explicit expression for the basic operators of the theory. Several attempts [11] have been made to simplify the Gauss law constraint (18), which in infinitesimal form reads $\hat{G}_{a}(x) \Phi_{A}(E)=0$, by means of a unitary transformation $\Phi_{A}^{\prime}(E)=$ $\exp \left\{-\frac{i}{r} \Omega(E)\right\} \Phi_{A}(E)$ in the electric field representation. The variation $\omega_{j}^{a}(E)=-\frac{\partial \Omega(E)}{\partial E_{a}^{j}}$ transforms as a standard Lie-algebra valued connection and modifies the operator $\hat{G}_{a}(x)$ so that the new constraint equations $\hat{G}_{a}^{\prime}(x) \Phi_{A}^{\prime}(E)=i C_{c}^{a b} E_{b} \cdot \frac{\delta}{\delta E_{c}} \Phi_{A}^{\prime}(E)=0$ reduce to simple "s-wave" conditions.

\section{b) The magnetic field representation $\Psi_{E}$.}

The choice of the polarization subalgebra $\mathcal{G}_{p}^{(E)}$ results in polarized wave functions of the form:

$$
\Psi_{E}(A, E, U ; \zeta)=\zeta e^{\frac{i}{r^{2}} \int d^{3} x \operatorname{tr}\left[A E-U \nabla U^{-1} E\right]} \Phi_{E}\left(A+\nabla U U^{-1}\right)
$$


where $\Phi_{E}$ is an arbitrary functional of $\mathcal{A} \equiv A+\nabla U U^{-1}$. The left-action of $\tilde{G}$ on wave functions $\Psi_{E}$ is now:

$$
\begin{aligned}
L_{\tilde{g}^{\prime}} \Psi_{E}(\tilde{g})= & \zeta^{\prime} \zeta e^{-2 \frac{i}{r^{2}} \int d^{3} x \operatorname{tr}\left[U^{\prime} A U^{\prime-1} E^{\prime}+U^{\prime-1} E^{\prime} U^{\prime} \nabla U U^{-1}+\frac{1}{2} A^{\prime} E^{\prime}-\frac{1}{2} U^{\prime} \nabla U^{\prime-1} E^{\prime}\right]} \\
& \cdot e^{-\frac{i}{r^{2}} \int d^{3} x \operatorname{tr}\left[A E-U \nabla U^{-1} E\right]} \Phi_{E}\left(A^{\prime}+\nabla U^{\prime} U^{\prime-1}+U^{\prime}\left(A+\nabla U U^{-1}\right) U^{\prime-1}\right)
\end{aligned}
$$

The constraint equations (18) in the present magnetic representation are:

$$
L_{\tilde{g}_{t}^{\prime}} \Psi(\tilde{g})=D_{\tilde{T}}^{(\epsilon)}\left(\tilde{g}_{t}^{\prime}\right) \Psi(\tilde{g}) \Rightarrow \Phi_{E}(\mathcal{A})=\Phi_{E}\left(U^{\prime} \mathcal{A} U^{\prime-1}+\nabla U^{\prime} U^{\prime-1}\right)
$$

[note the absence of a phase in comparison with the electric representation case]. The infinitesimal form of the finite expression (21) is:

$$
\begin{aligned}
\tilde{X}_{A_{a}}^{R} \Psi_{E} & =\zeta e^{\frac{i}{r^{2}} \int d^{3} x \operatorname{tr}\left[A E-U \nabla U^{-1} E\right]} \frac{\delta}{\delta \mathcal{A}_{a}} \Phi_{E}(\mathcal{A}) \Rightarrow \hat{E}_{a} \Phi_{E}(\mathcal{A})=i \frac{\delta}{\delta \mathcal{A}_{a}} \Phi_{E}(\mathcal{A}) \\
\tilde{X}_{E_{a}}^{R} \Psi_{E} & =-i \zeta e^{\frac{i}{r^{2}} \int d^{3} x \operatorname{tr}\left[A E-U \nabla U^{-1} E\right]} \mathcal{A}_{a} \Phi_{E}(\mathcal{A}) \Rightarrow \hat{A}_{a} \Phi_{E}(\mathcal{A})=\mathcal{A}_{a} \Phi_{E}(\mathcal{A}) \\
\tilde{X}_{\varphi_{a}}^{R} \Psi_{E} & =\zeta e^{\frac{i}{r^{2}} \int d^{3} x \operatorname{tr}\left[A E-U \nabla U^{-1} E\right]}\left(-\frac{1}{r} \nabla \cdot \frac{\delta}{\delta \mathcal{A}_{a}}+C_{c}^{a b} \mathcal{A}_{b} \cdot \frac{\delta}{\delta \mathcal{A}_{c}}\right) \Phi_{E}(\mathcal{A})
\end{aligned}
$$

Since $\tilde{T}$-equivariant conditions $(8,18,22)$ are imposed as finite left-restrictions, it is evident that not all the operators $\tilde{X}^{R}$ will preserve the constraints; we shall call $\tilde{\mathcal{G}}_{\text {good }}$ the subalgebra of (good physical) operators which will do so. These must be found inside the right-enveloping algebra $\mathcal{U}\left(\tilde{\mathcal{G}}^{R}\right)$ of polynomials of the basic operators $\hat{A}_{a}(x), \hat{E}_{b}(x)$, as forming part of the normalizer of $T$; for example, a sufficient condition for $\tilde{\mathcal{G}}_{\text {good }}$ to preserve the constraints is $\left[\tilde{\mathcal{G}}_{\text {good }}, \tilde{\mathcal{T}}\right] \subset \operatorname{Ker} d D_{\tilde{T}}^{(\epsilon)}$. In particular, some good operators are:

$$
\tilde{\mathcal{G}}_{\text {good }}=<\operatorname{tr}\left[\hat{E}^{j}(x) \hat{B}^{k}(x)\right], \operatorname{tr}\left[\hat{E}^{j}(x) \hat{E}^{k}(x)\right], \operatorname{tr}\left[\hat{B}^{j}(x) \hat{B}^{k}(x)\right], \Xi>,
$$

where $\hat{B}_{a} \equiv \nabla \wedge \hat{A}_{a}-\frac{1}{2} r C_{c}^{a b} \hat{A}_{b} \wedge \hat{A}_{c}$ (the magnetic field) can be interpreted as a "correction" to $\hat{A}_{a}$ that, unlike $\hat{A}_{a}$, transforms homogeneously under the adjoint action of $T$ [see 2nd line of (13)]. The components $\hat{\Theta}^{\mu \nu}(x)$ of the standard canonical energy-momentum tensor for Yang-Mills theories are linear combinations of operators in (24); for example, $\hat{\Theta}^{00}(x)=-\operatorname{tr}\left[\hat{E}^{2}(x)+\hat{B}^{2}(x)\right]$ is the Hamiltonian density. In this way, Poincaré invariance is retrieved in the constrained theory. At this stage, it is worth mentioning that $\mathcal{G}_{c}$ would have included the entire Poincaré algebra had we incorporated the Poincaré group into $\tilde{G}$ (see $[4,5]$ for the Abelian case). However, unlike other standard approaches to Quantum Mechanics, GAQ still remainss even in the absence of a well-defined (space)time evolution, an interesting and desirable property concerning the quantization of gravity (see, for example, [12]).

Let us mention, for the sake of completeness, that the actual use of good operators is not restricted to first- and second-order operators. Higher-order operators can constitute a useful tool in finding the whole constrained Hilbert space $\mathcal{H}_{\text {phys }}(\tilde{G})$. In fact, it can be obtained from a $\tilde{T}$-equivariant (physical) state $\Phi^{(0)}$, i.e. $\hat{G}_{a} \Phi^{(0)}=0$, on which the 
energy-momentum tensor has null expectation value $\left\langle\Phi^{(0)}\left|\hat{\Theta}^{\mu \nu}\right| \Phi^{(0)}\right\rangle=0$, by taking the orbit of the rest of good operators passing through this "vacuum". This has indeed been a rather standard technique (the Verma module approach) in theories where null vector states are present in the original Hilbert space $[13,14,10]$. From another point of view, with regard to confinement, exponentials of the form $\varepsilon_{\Sigma_{2}} \equiv \operatorname{tr}\left[\exp \left(\epsilon_{j k l} \int_{\Sigma_{2}} d \sigma^{j k} \hat{E}^{l}\right)\right]$ and $\beta_{\Sigma_{2}} \equiv \operatorname{tr}\left[\exp \left(\epsilon_{j k l} \int_{\Sigma_{2}} d \sigma^{j k} \hat{B}^{l}\right)\right]$, where $\Sigma_{2}$ is a two-dimensional surface in three-dimensional space, are good operators related to Wilson loops.

As a step prior to tackling the massive case, let us show how new physics can enter the theory by considering non-trivial representations $D_{\tilde{T}}^{(\epsilon)}$ of $\tilde{T}$ or, in an equivalent way, by introducing certain extra pseudo-cocycles in the group law (10).

\section{$3.1 \quad \vartheta$-Angle}

More general representations for the constraint subgroup $T$, namely the one-dimensional representation $D_{\tilde{T}}^{(\epsilon)}(U)=e^{i \epsilon_{U}}$, can be considered if we impose additional boundary conditions such as $U(x) \stackrel{x \rightarrow \infty}{\longrightarrow} \pm I$; this means that we compactify the space $\Re^{3} \rightarrow S^{3}$, so that the group $T$ falls into disjoint homotopy classes $\left\{U_{l}, \epsilon_{U_{l}}=l \vartheta\right\}$ labeled by integers $l \in Z=\pi_{3}(\mathbf{T})$ (the third homotopy group). The index $\vartheta$ (the $\vartheta$-angle [7]) parametrizes non-equivalent quantizations, in the same way that Bloch momentum $\epsilon$ does for particles in periodic potentials, where the wave function acquires a phase $\psi(q+2 \pi)=e^{i \epsilon} \psi(q)$ after a translation of, let us say, $2 \pi$. The phenomenon of non-equivalent quantizations can also be reproduced by keeping the constraint condition $D_{\tilde{T}}^{(\epsilon)}(U)=1$, as in $(18,22)$, at the expense of introducing a new cocycle (indeed a coboundary) $\xi_{\vartheta}$ which is added to the previous cocycle $\xi$ in (10). The generating function of $\xi_{\vartheta}$ is $\eta_{\vartheta}(g)=\vartheta \int d^{3} x \mathcal{C}^{0}(x)$, where $\mathcal{C}^{0}$ is the time component of the Chern-Simons secondary characteristic class

$$
\mathcal{C}^{\mu}=-\frac{1}{16 \pi^{2}} \epsilon^{\mu \alpha \beta \gamma} \operatorname{tr}\left(\mathcal{F}_{\alpha \beta} \mathcal{A}_{\gamma}-\frac{2}{3} \mathcal{A}_{\alpha} \mathcal{A}_{\beta} \mathcal{A}_{\gamma}\right)
$$

which is the vector whose divergence equals the Pontryagin density $\mathcal{P}=\partial_{\mu} \mathcal{C}^{\mu}=-\frac{1}{16 \pi^{2}} \operatorname{tr}$ $\left({ }^{*} \mathcal{F}^{\mu \nu} \mathcal{F}_{\mu \nu}\right)$ (see [8], for instance). Like some total derivatives (namely, the Pontryagin density), which do not modify the classical equations of motion when added to the Lagrangian but have a non-trivial effect in the quantum theory, the coboundary $\xi_{\vartheta}$ gives rise to nonequivalent quantizations parametrized by $\vartheta$ when the topology of the space is affected by the imposition of certain boundary conditions ("compactification of the space"), even though it is a trivial cocycle of the "unconstrained" theory. The phenomenon of nonequivalent quantizations can sometimes also be understood as an Aharonov-Bohm-like effect (an effect experienced by the quantum particle but not by the classical one) and the gradient $d \eta(g)$ can also be understood as an induced gauge connection (see e.g. [15, 16], and [17] for the example of a superconducting ring threaded by a magnetic flux) which modifies momenta according to the minimal coupling. For our case, the induced gauge connection $\delta \eta_{\vartheta}(g)=\frac{\vartheta r^{2}}{8 \pi^{2}} B_{j}^{a} \delta A_{a}^{j}$ ( $B_{j}^{a}$ is the magnetic field) modifies the momentum operators $\hat{E}_{a} \equiv i \tilde{X}_{A_{a}}^{R} \rightarrow \hat{E}_{a}+\frac{\vartheta r^{2}}{8 \pi^{2}} \hat{B}_{a}$ and, accordingly, the Schrödinger equation $\int d^{3} x \hat{\Theta}^{00}(x) \Phi=\mathcal{E} \Phi$ 
for stationary solutions $\Phi$ with energy $\mathcal{E}$. As is well known, the theory also exhibits a band energy structure of the form $\alpha+\beta \cos \vartheta$, the ground-state band functional $|\vartheta\rangle=\sum_{l} e^{i l \vartheta}\left|0_{l}\right\rangle$ being a superposition of wave functionals $\Psi_{l}(A)=\left\langle A \mid 0_{l}\right\rangle$ peaked near the classical zeroenergy configurations (pure gauge potentials) $A_{(l)}=U_{l} \nabla U_{l}^{-1}$.

As already discussed, only coboundaries generated by functions $\eta(g)$ with non-trivial gradient $\left.\delta \eta(g)\right|_{g=e} \neq 0$ at the identity $g=e$ (i.e. pseudo-cocycles), namely $\xi_{3}=\xi_{\lambda}$, will provide a contribution to the connection form of the theory (11) and the structure constants of the original Lie algebra. However, as we have just seen, a coboundary generated by a global function on the original (infinite-dimensional) group $G$ having trivial gradient at the identity, namely $\xi_{\vartheta}$, can contribute the quantization with global (topological) effects as the new group has a non-equivalent global multiplication law.

In both cases, non-trivial gauge transformation properties, $D_{\tilde{T}}^{(\epsilon)}(U) \neq 1$, of the wave functional $\Phi(A)$ can be reproduced, as already mentioned, by keeping the trivial representation $D_{\widetilde{T}}^{(\epsilon)}(U)=1$ at the expense of introducing new (pseudo-) cocycles, $\xi_{\vartheta}$ or $\xi_{\lambda}$, in the centrally extended group law (10). However, whereas $\xi_{\vartheta}$ does not introduce new degrees of freedom into the theory, pseudo-cocycles such as $\xi_{\lambda}$ provide new couples of conjugated field operators, thus substantially modifying the theory. Let us examine this in more detail.

\section{The massive case: 'spontaneous' symmetry 'break- ing' and alternatives to the Higgs mechanism}

The effect of the pseudo-cocycle $\xi_{3} \equiv \xi_{\lambda}$ for $\lambda \neq 0$ is equivalent to inducing internal ('spinor-like') infinite-dimensional non-Abelian representations $D_{\tilde{T}}^{(\lambda)}$ of $\tilde{T}$. It modifies the commutation relations (13) and the number of field degrees of freedom of the theory by restricting the number of vector fields in the characteristic subalgebra $\mathcal{G}_{c}$ with respect to the massless case, where $\mathcal{G}_{c} \sim \mathcal{T}$. That is, new couples of generators $\left(\tilde{X}_{\varphi_{a}}^{R}, \tilde{X}_{\varphi_{b}}^{R}\right)$, with $C_{c}^{a b} \lambda^{c} \neq 0$, become conjugated [see the last commutator of (13)] and, therefore, new basic operators enter the theory. To count the number of field degrees of freedom for a given structure subgroup $\tilde{T}$ and a given mass matrix $\lambda=\lambda_{a} T^{a}$, let us denote by $\tau=\operatorname{dim}(\mathbf{T})$ and $c=\operatorname{dim}\left(\mathbf{G}_{c}\right)$ the dimensions of the rigid subgroups of $T$ and $G_{c}$; in general, for an arbitrary mass matrix $\lambda$, we have $c \leq \tau$. Unpolarized, $U(1)$-equivariant functions $\Psi\left(A_{a}^{j}, E_{a}^{j}, \varphi_{a}\right)$ depend on $n=2 \times 3 \tau+\tau$ field coordinates in $d=3$ dimensions; polarization equations introduce $p=c+\frac{n-c}{2}$ independent restrictions on wave functions, corresponding to $c$ non-dynamical coordinates in $G_{c}$ and half of the dynamical ones; finally, constraints impose $q=c+\frac{\tau-c}{2}$ additional restrictions which leave $f=n-p-q=3 \tau-c$ field degrees of freedom (in $d=3$ ). Indeed, for the massive case, constraints are second-class and we can impose only a polarization subalgebra $\mathcal{T}_{p} \subset \tilde{\mathcal{T}}$, which contains a characteristic subalgebra $\mathcal{T}_{c}=<\tilde{X}_{\varphi_{a}}^{R}$, with $C_{c}^{a b} \lambda^{c}=0 \forall b>\subset \tilde{\mathcal{T}}$ (which is isomorphic to $\mathcal{G}_{c}$ ) and half of the rest 
of generators in $\tilde{\mathcal{T}}$ (excluding $\Xi$ ) $;^{\S}$ In total, $q=c+\frac{\tau-c}{2} \leq \tau$ independent constraints, which lead to constrained wave functions having support on $f_{m \neq 0}=3 \tau-c \geq f_{m=0}$ arbitrary fields; these fiels correspond to $c$ massless vector bosons attached to $\mathcal{T}_{c}$ and $\tau-c$ massive vector bosons. In particular, for the massless case, we have $\mathcal{T}_{c}=\mathcal{T}$, i.e. $c=\tau$, since constraints are first-class (that is, we can impose $q=\tau$ restrictions) and constrained wave functions have support on $f_{m=0}=3 \tau-\tau=2 \tau \leq f_{m \neq 0}$ arbitrary fields corresponding to $\tau$ massless vector bosons. The subalgebra $\mathcal{T}_{c}$ corresponds to the unbroken gauge symmetry of the constrained theory and proves to be an ideal of $\tilde{\mathcal{G}}_{\text {good }}$ [remember the characterization of good operators before Eq. (24); see also Refs. [2, 5] for a definition and subtle distinctions between constraints and gauge symmetries inside GAQ].

Let us work out a couple of examples. Cartan (maximal Abelian) subalgebras of $\mathbf{T}$ will be preferred as candidates for the rigid subgroup of the unbroken electromagnetic gauge symmetry. Thus, let us use the Cartan basis $\left\langle H_{i}, E_{ \pm \alpha}\right\rangle$ instead of $\left\langle T^{a}\right\rangle$, and denote $\left\{\varphi_{i}, \varphi_{ \pm \alpha}\right\}$ the coordinates of $T$ attached to this basis (i.e. $\varphi_{ \pm \alpha}$ are complex field coordinates attached to each root $\pm \alpha$, and $\varphi_{i}$ are real field coordinates attached to the maximal torus of $\mathbf{T})$. For $T=S U(2)(x)$ and $\lambda=\lambda_{1} H_{1}$, the characteristic, polarization and constraint subalgebras (leading to the electric field representation) are:

$$
\mathcal{G}_{c}=<\tilde{X}_{\theta_{1}}^{L}>, \mathcal{G}_{p}^{(A)}=<\tilde{X}_{\theta_{1}}^{L}, \tilde{X}_{\theta_{+1}}^{L}, \tilde{X}_{A}^{L}>, \mathcal{T}_{p}=<\tilde{X}_{\varphi_{1}}^{R}, \tilde{X}_{\varphi_{-1}}^{R}>
$$

Indeed, the appearance of a central term in the commutator

$$
\left[\tilde{X}_{\varphi_{+1}(x)}^{R}, \tilde{X}_{\varphi_{-1}(y)}^{R}\right]=i \delta(x-y) \tilde{X}_{\varphi_{1}(x)}^{R}+i \frac{\lambda_{1}}{r^{2}} \delta(x-y) \Xi
$$

prevents the vector fields $\tilde{X}_{\theta_{ \pm 1}}^{R}$ from being in $\mathcal{G}_{c}$ and precludes the simultaneous imposition of $\tilde{X}_{\varphi_{-1}}^{R} \Psi_{\text {phys. }}=0$ and $\tilde{X}_{\varphi+1}^{R} \Psi_{\text {phys. }}=0$ as constraints (for the trivial representation $\left.D_{\tilde{T}}^{(\epsilon)}(U)=1\right)$, so that a polarization subalgebra $\mathcal{T}_{p}$ is the only option $\left(\mathcal{T}_{p}\right.$ has to contain the 'negative modes' $\tilde{X}_{\varphi_{-1}}^{R}$ when the 'positive' ones $\tilde{X}_{\theta_{+1}}^{L}$ have been chosen in $\mathcal{G}_{p}^{(A)}$, or the other way round). The new couple of basic operators $\hat{G}_{ \pm 1} \equiv \tilde{X}_{\varphi_{ \pm 1}}^{R}$ (these are basic because they can no longer be written in terms of $\hat{A}$ and $\hat{E}$ ) represent two new field degrees of freedom which are transferred to the vector potentials $\hat{A}_{ \pm 1}$ to conform massive vector bosons; i.e. $\hat{G}_{ \pm 1}$ can be seen as the longitudinal component of $\hat{A}_{ \pm 1}$, which is missing (is zero) in the massless case. Thus, the constrained theory corresponds to a self-interacting field theory of a massless vector boson $A_{1}$ with 'unbroken' gauge subgroup $T_{c}=U(1)(x) \subset S U(2)(x)$ and two charged vector bosons $A_{ \pm 1}$ with mass cubed $m_{1}^{3}=\lambda_{1}$.

For $T=S U(3)(x)$ and $\lambda=\lambda_{2} H_{2}$, we have

$$
\begin{gathered}
\mathcal{G}_{c}=<\tilde{X}_{\theta_{1,2}}^{L}, \tilde{X}_{\theta_{ \pm 1}}^{L}>, \mathcal{G}_{p}^{(A)}=<\tilde{X}_{\theta_{1,2}}^{L}, \tilde{X}_{\theta_{ \pm 1}}^{L}, \tilde{X}_{\theta_{+2,+3}}^{L}, \tilde{X}_{A}^{L}>, \\
\mathcal{T}_{p}=<\tilde{X}_{\varphi_{1,2}}^{R}, \tilde{X}_{\varphi_{ \pm 1}}^{R}, \tilde{X}_{\varphi_{-2,-3}}^{R}>
\end{gathered}
$$

${ }^{\S} \mathrm{A}$ similar situation happens in the bosonic string theory, where we can impose as constraints half of the Virasoro operators (the positive modes $\hat{L}_{n \geq 0}$ ) only; that is, the appearance of central terms in the Lie algebra (12) precludes the whole Virasoro algebra to be imposed as constraints, and only a polarization subalgebra can be consistently imposed. 
Indeed, in this case, the relevant commutators

$$
\begin{aligned}
{\left[\tilde{X}_{\varphi_{+1}(x)}^{R}, \tilde{X}_{\varphi_{-1}(y)}^{R}\right] } & =i \delta(x-y) \tilde{X}_{\varphi_{1}(x)}^{R}, \\
{\left[\tilde{X}_{\varphi_{+2}(x)}^{R}, \tilde{X}_{\varphi_{-2}(y)}^{R}\right] } & =\frac{i}{\sqrt{3}} \delta(x-y) \tilde{X}_{\varphi_{1}(x)}^{R}+i \delta(x-y) \tilde{X}_{\varphi_{2}(x)}^{R}+i \frac{\lambda_{2}}{r^{2}} \delta(x-y) \Xi, \\
{\left[\tilde{X}_{\varphi_{+3}(x)}^{R}, \tilde{X}_{\varphi_{-3}(y)}^{R}\right] } & =\frac{-i}{\sqrt{3}} \delta(x-y) \tilde{X}_{\varphi_{1}(x)}^{R}+i \delta(x-y) \tilde{X}_{\varphi_{2}(x)}^{R}+i \frac{\lambda_{2}}{r^{2}} \delta(x-y) \Xi,
\end{aligned}
$$

reveal that the vector fields $\tilde{X}_{\theta_{ \pm 2}}^{R}$ and $\tilde{X}_{\theta_{ \pm 3}}^{R}$ have dynamical content and cannot be included in $\mathcal{G}_{c}$. Also, its conjugated character precludes the simultaneous imposition of $\tilde{X}_{\varphi_{-2,-3}}^{R}$ and $\tilde{X}_{\varphi+2,+3}^{R}$ as constraints, and a polarization subalgebra $\mathcal{T}_{p}$ has to be chosen. On the contrary, the vector fields $\tilde{X}_{\varphi_{ \pm 1}}^{R}$ are devoid of dynamical content, as can be seen from the first line of (29), and can be simultaneously imposed as constraints in $\mathcal{T}_{p}$ (this is because of the particular choice of mass matrix $\lambda$, which determines different "symmetry breaking" patterns). As for $T=S U(2)(x)$, the new couples of basic operators $\hat{G}_{ \pm 2, \pm 3} \equiv \tilde{X}_{\varphi_{ \pm 2, \pm 3}}^{R}$ represent four new field degrees of freedom which are transferred to the vector potentials $\hat{A}_{ \pm 2, \pm 3}$ to conform massive vector bosons. Thus, the constrained theory corresponds to a self-interacting theory of two massless vector bosons $A_{1,2}$, two massless charged vector bosons $A_{ \pm 1}$ [the 'unbroken' gauge subgroup is now $T_{c}=S U(2) \times U(1)(x) \subset S U(3)(x)$ ] and four charged vector bosons $A_{ \pm 2, \pm 3}$ with mass cubed $m_{2}^{3}=\lambda_{2}$.

Summarizing, new basic operators $\hat{G}_{ \pm \alpha} \equiv \tilde{X}_{\varphi_{ \pm \alpha}}^{R}$, with $C_{i}^{\alpha-\alpha} \lambda^{i} \neq 0$, and new good operators $\hat{C}_{i}=\{$ Casimir operators of $\tilde{T}\}$ ( $i$ runs the range of $\mathbf{T}$ ) enter the theory, in contrast to the massless case. For example, for $T=S U(2)(x)$, the Casimir operator is

$$
\hat{C}(x)=\left(\hat{G}_{1}(x)+\frac{\lambda_{1}}{r^{2}}\right)^{2}+2\left(\hat{G}_{+1}(x) \hat{G}_{-1}(x)+\hat{G}_{-1}(x) \hat{G}_{+1}(x)\right) .
$$

Also, the Hamiltonian density $\hat{\Theta}^{00}(x)=-\operatorname{tr}\left[E^{2}(x)+B^{2}(x)\right]$ for $m=0$ can be affected in the massive case $m \neq 0$ by the presence of extra terms proportional to these Casimir operators as follows:

$$
\hat{\Theta}_{m \neq 0}^{00}(x)=\hat{\Theta}_{m=0}^{00}(x)+\sum_{i} \frac{r^{2}}{m_{i}^{2}} \hat{C}_{i}(x) .
$$

Thus, the Schödinger equation $\int d^{3} x \hat{\Theta}_{m \neq 0}^{00}(x) \Phi=\mathcal{E} \Phi$ is also modified by the presence of extra terms.

As already mentioned in reference to the Virasoro group, pseudo-cocycle parameters such as $\lambda_{i}$ are usually hidden in a redefinition of the generators involved in the pseudo-extension $\hat{G}_{i}(x)+\lambda_{i} / r^{2} \equiv \hat{G}_{i}^{\prime}(x)$. However, whereas the vacuum expectation value $\left\langle 0_{\lambda}\left|\hat{G}_{i}(x)\right| 0_{\lambda}\right\rangle$ is zero, " the vacuum expectation value $\left\langle 0_{\lambda}\left|\hat{G}_{i}^{\prime}(x)\right| 0_{\lambda}\right\rangle=\lambda_{i} / r^{2}$ of the redefined operators $\hat{G}_{i}^{\prime}(x)$ is non-null and proportional to the cubed mass in the 'direction' $i$ of the 'unbroken' gauge symmetry $T_{c}$, which depends on the particular choice of

\footnotetext{
$\boldsymbol{I}_{\text {it }}$ can be easily proven taking into account that the vacuum is annihilated by the right version of the polarization subalgebra dual to $\mathcal{G}_{p}[18]$; also, $\hat{G}_{i}=\tilde{X}_{\varphi_{i}}^{R}$ is always in $\mathcal{T}_{p}$; that is, it is zero on constrained wave functionals $\Psi_{\text {phys. }}$, including the physical vacuum.
} 
the mass matrix $\lambda$. Thus, the effect of the pseudo-extension manifests also in a different choice of a vacuum in which some gauge operators have a non-zero expectation value. This fact reminds us of the Higgs mechanism in non-Abelian gauge theories, where the Higgs fields point to the direction of the non-null vacuum expectation values. However, the spirit of the Higgs mechanism, as an approach to supply mass, and the one discussed in this paper are radically different, even though they have some common characteristics. In fact, we are not making use of extra scalar fields in the theory to provide mass to the vector bosons, but it is the gauge group itself that acquires dynamics for the massive case and transfers degrees of freedom to the vector potentials.

Before finishing, let us show how to incorporate fermionic matter into the theory and outline the main changes in the foregoing discussion had we considered it from the beginning.

\section{Incorporating fermionic matter}

Fermionic matter can enter the theory through extra (Dirac) field coordinates $\psi_{l}(x), l=$ $1, \ldots, p$, which we can assemble into a column vector $\psi(x)$, and an extra cocycle $\xi_{\text {matter }}$ leading to a quantizing supergroup $\widetilde{S G}$. The group law that describes this boson-fermion gauge theory is (10) together with

$$
\begin{aligned}
\psi^{\prime \prime}(x) & =\psi^{\prime}(x)+\rho(U(x)) \psi(x) \\
\bar{\psi}^{\prime \prime}(x) & =\bar{\psi}^{\prime}(x)+\bar{\psi}(x) \rho\left(U(x)^{-1}\right) \\
\xi_{\text {matter }} & \equiv i \int d^{3} x\left(\bar{\psi}^{\prime} \gamma^{0} \rho\left(U^{\prime}\right) \psi-\bar{\psi} \rho\left(U^{\prime-1}\right) \gamma^{0} \psi^{\prime}\right)
\end{aligned}
$$

where $\rho(U)$ is a $p$-dimensional representation of $\mathbf{T}$ acting on the column vectors $\psi$, and $\gamma^{0}$ is the time component of the standard Dirac matrices $\gamma^{\mu}$. To compute the left- and right-invariant super-vector fields $\tilde{X}^{L, R}$ and the polarized super-wavefunctionals $\Psi(A, E, U, \psi, \bar{\psi} ; \zeta)$, we have to take into account the Grassmann character of the Dirac field coordinates. The unitary irreducible representations of $\widetilde{S G}$ can easily be constructed by following the main steps described in this article and by taking care of the subtleties introduced by the anti-commutation of Grassmann variables (see [19] for the finite-dimensional

example of the super-Galilei group $\left.\widetilde{S G}_{(m)}\right)$. We should mention that, in the presence of fermion sources, the infinitesimal version of the constraint (18), i.e. the Gauss law, is modified to

$$
\hat{G}_{a} \Phi_{A, \psi}(E, \bar{\psi})=\left(-\frac{1}{r} \nabla \cdot \hat{E}_{a}-C_{c}^{a b} \hat{E}_{b} \cdot \hat{A}_{c}-\frac{i}{r} \hat{\bar{\psi}} \gamma^{0} \tau_{a} \hat{\psi}\right) \Phi_{A, \psi}(E, \bar{\psi})=0,
$$

(where $\tau_{a}$ denote the generators of $\rho$ ) in accordance with other standard approaches. Other interesting questions like chiral anomalies are left to future publications. 


\section{Some comments and outlook}

One question which is worthwhile to comment upon is the preservation of renormalizability for a non-trivial mass matrix $\lambda \neq 0$. Since our approach to quantization is not perturbative, we must answer this question using general arguments. In fact, from a group-theoretical point of view, there is no reason why a given unitary irreducible representation of a group $\tilde{G}$ (namely, the massive one) can show bad properties, like 'inescapable divergences', whereas other (namely, the massless one) does not. Even more, when we use the term 'unbroken gauge symmetry', in referring to $T_{c}$, we mean simply the subgroup of $\tilde{T}$ devoid of dynamical content; the gauge group of the constrained theory is, in both the massless and massive cases, the group $T=\tilde{T} / U(1)$, although, for the massive case, only a polarization subgroup $T_{p}$ can be consistently imposed as a constraint. This is also the case of the Virasoro algebra (12) in String Theory, where the appearance of central terms does not spoil gauge invariance but forces us to impose half of the Virasoro operators only (the positive modes $\hat{L}_{n \geq 0}$ ) as constraints.

Thus, the 'spontaneous breakdown' of the gauge symmetry group $T$ manifests through non-trivial transformations of the phase $\zeta$ of the wave functional $\Psi$ under the action of $T$, leading to the appearance of new 'internal' field degrees of freedom which modify the 'field mass content' of some vector potentials $A$, depending on the choice of mass-matrix elements $\lambda^{i}=-2 \operatorname{tr}\left(T^{i} \lambda\right)$. This situation recalls the important physical implications of geometric phases (namely, Berry's phase) in quantum mechanics, the case discussed in the present paper being a particular one. In other words, the presence of mass is detected by the wave functional $\Psi$ in its 'gauge excursions' through the configuration space, as happens with the presence of monopoles (see Ref. [16, 15] for a discussion on the emergence of gauge structures - the " $H$-connection" - and generalized spin when quantizing on a coset space $G / H)$. Also, the zeroes (critical values) of the mass-matrix elements $\lambda^{i}$ correspond to different phases of the physical system characterized by the corresponding unbroken gauge symmetry $T_{c}$; thus, the system can undergo 'spontaneous' phase-transitions between different phases corresponding to non-equivalent fibrations $\tilde{T}$ of $T$ by $U(1)$ (i.e. different choices of characteristic subgroups $T_{c}$ of $\tilde{T}$ ).

Open questions remain about what happens when a "true" cocycle $\xi_{3}$ exists; for example, we can find non-trivial central extensions $\tilde{T}$ of $T=\operatorname{Map}\left(S^{1}, \mathbf{T}\right)$ by $U(1)$ (Kac-Moody groups) in one compact spatial dimension, deformations which correspond to anomalous situations in the standard (canonical) approach to quantization of gauge theories. This fact makes the quantization of 'massive' Yang-Mills fields (in this scheme) not so trivial, even in one spatial dimension. Also, it would be worth exploring the richness of the case $\mathbf{T}=S U(\infty)$ (infinite number of colours), the Lie-algebra of which is related to the (infinite-dimensional) Lie-algebra of area preserving diffeomorphisms of the sphere $S \operatorname{Diff}\left(S^{2}\right)$ (see [20] and references therein). In general, the cohomological richness, i.e. the number of inequivalent central (pseudo) extensions, of $T=\operatorname{Map}(M, \mathbf{T})$ depends on the topology of $M$. Also, as usually happens with central charges, a quantization of the

mass parameters $m_{c} \sim(n)^{1 / 3}, n=1,2,3, \ldots$ could arise from the compact character of the involved manifolds. 
Another question that deserves further study is, of course, the physical implications that this new point of view carries along.

\section{A Appendix: a $0+1 \mathrm{D}$ analogy}

This appendix is intended to clarify ideas by providing a simple, but illustrative, quantum mechanical analogy which contains most of the essential elements exposed in the paper. Indeed, a $S U(2)$ gauge invariant Yang-Mills theory in $0+1 \mathrm{D}$ may eventually be related to a spinning particle with constraints (zero total angular momentum) inside the present GAQ framework.

Let us denote by $A \equiv r\left(\begin{array}{cc}A_{0} & A_{+} \\ A_{-} & -A_{0}\end{array}\right), A_{0} \equiv A_{3}, A_{ \pm} \equiv A_{1} \pm i A_{2}$, the $s u(2)$-valued vector potentials, and let us choose the following set of coordinates

$$
e^{i \varphi_{0}} \equiv \frac{z_{1}}{\left|z_{1}\right|}, \quad \varphi_{+} \equiv \frac{z_{2}}{z_{1}}, \quad \varphi_{-} \equiv \frac{z_{2}^{*}}{z_{1}^{*}}, \quad e^{i \varphi_{0}} \in S^{1} ; \quad \varphi_{+}, \varphi_{-} \in S^{2}
$$

for the gauge group

$$
S U(2) \equiv\left\{U=\left(\begin{array}{cc}
z_{1} & z_{2} \\
-z_{2}^{*} & z_{1}^{*}
\end{array}\right), z_{i}, z_{i}^{*} \in C / \operatorname{det}(U)=\left|z_{1}\right|^{2}+\left|z_{2}\right|^{2}=1\right\} .
$$

Let us also work in an holomorphic picture and define $Q \equiv \frac{1}{\sqrt{2} r}(A+i E)$ and $\bar{Q} \equiv$ $\frac{1}{\sqrt{2} r}(A-i E)$. The adjoint action of the gauge group on the vector potential $A$ and the electric field $E$ can be explicitly written as

$$
U Q U^{-1}=\frac{1}{1+\varphi_{+} \varphi_{-}}\left(\begin{array}{cc}
e^{i \varphi_{0}} & \varphi_{+} e^{i \varphi_{0}} \\
-\varphi_{-} e^{-i \varphi_{0}} & e^{-i \varphi_{0}}
\end{array}\right)\left(\begin{array}{cc}
Q_{0} & Q_{+} \\
Q_{-} & -Q_{0}
\end{array}\right)\left(\begin{array}{cc}
e^{-i \varphi_{0}} & -\varphi_{+} e^{i \varphi_{0}} \\
\varphi_{-} e^{-i \varphi_{0}} & e^{i \varphi_{0}}
\end{array}\right)
$$

and the centrally extended group law (10) now adopts the form

$$
\begin{aligned}
& U^{\prime \prime}=U^{\prime} U \\
& Q^{\prime \prime}=Q+U^{-1} Q^{\prime} U \text {, } \\
& \bar{Q}^{\prime \prime}=\bar{Q}+U^{-1} \bar{Q}^{\prime} U \text {, } \\
& \zeta^{\prime \prime}=\zeta^{\prime} \zeta \exp \frac{1}{4} \operatorname{tr}\left[\left(\begin{array}{ll}
Q & \bar{Q}
\end{array}\right) S\left(\begin{array}{c}
U^{-1} Q^{\prime} U \\
U^{-1} \bar{Q}^{\prime} U
\end{array}\right)\right] \exp 2 i \lambda\left(\varphi_{0}^{\prime \prime}-\varphi_{0}^{\prime}-\varphi_{0}\right),
\end{aligned}
$$

where we miss the mixed cocycle $\xi_{2}$ because we are working in 0 spatial dimensions (we are restricting ourselves to a "single point"). We are also keeping only the (relevant) linear term $\lambda \varphi_{0}$ in the expansion of $\operatorname{tr}\left[\lambda \sigma_{3} \log U\right]\left(\sigma_{3}\right.$ is the standard Pauli matrix). The left- and right-invariant vector fields are explicitly:

$$
\tilde{X}_{\zeta}^{L}=\tilde{X}_{\zeta}^{R}=\zeta \frac{\partial}{\partial \zeta}
$$




$$
\begin{aligned}
\tilde{X}_{Q}^{L} & =\frac{\partial}{\partial Q}+\frac{1}{2} \bar{Q} \zeta \frac{\partial}{\partial \zeta}, \quad \tilde{X}_{\bar{Q}}^{L}=\frac{\partial}{\partial \bar{Q}}-\frac{1}{2} Q \zeta \frac{\partial}{\partial \zeta} \\
\tilde{X}_{\varphi_{0}}^{L} & =\frac{\partial}{\partial \varphi_{0}}-2 i \varphi_{+} \frac{\partial}{\partial \varphi_{+}}+2 i \varphi_{-} \frac{\partial}{\partial \varphi_{-}}-2\left(Q \times \frac{\partial}{\partial Q}\right)_{0}-2\left(\bar{Q} \times \frac{\partial}{\partial \bar{Q}}\right)_{0} \\
\tilde{X}_{\varphi_{+}}^{L} & =\frac{-i}{2} \varphi_{-} \frac{\partial}{\partial \varphi_{0}}+\frac{\partial}{\partial \varphi_{+}}+\varphi_{-}^{2} \frac{\partial}{\partial \varphi_{-}}+i\left(Q \times \frac{\partial}{\partial Q}\right)_{-}+i\left(\bar{Q} \times \frac{\partial}{\partial \bar{Q}}\right)_{-}+\lambda \varphi_{-} \zeta \frac{\partial}{\partial \zeta} \\
\tilde{X}_{\varphi_{-}}^{L} & =\frac{i}{2} \varphi_{+} \frac{\partial}{\partial \varphi_{0}}+\varphi_{+}^{2} \frac{\partial}{\partial \varphi_{+}}+\frac{\partial}{\partial \varphi_{-}}-i\left(Q \times \frac{\partial}{\partial Q}\right)_{+}-i\left(\bar{Q} \times \frac{\partial}{\partial \bar{Q}}\right)_{+}-\lambda \varphi_{+} \zeta \frac{\partial}{\partial \zeta}, \\
\tilde{X}_{Q}^{R} & =U \frac{\partial}{\partial Q} U^{-1}-\frac{1}{2} U \bar{Q} U^{-1} \zeta \frac{\partial}{\partial \zeta}, \\
\tilde{X}_{\bar{Q}}^{R} & =U \frac{\partial}{\partial \bar{Q}} U^{-1}+\frac{1}{2} U Q U^{-1} \zeta \frac{\partial}{\partial \zeta}, \\
\tilde{X}_{\varphi_{0}}^{R} & =\frac{\partial}{\partial \varphi_{0}}, \\
\tilde{X}_{\varphi_{+}}^{R} & =\frac{i}{2} e^{-2 i \varphi_{0}} \varphi_{-} \frac{\partial}{\partial \varphi_{0}}+e^{-2 i \varphi_{0}}\left(1+\varphi_{+} \varphi_{-}\right) \frac{\partial}{\partial \varphi_{+}}-\lambda e^{-2 i \varphi_{0}} \varphi_{-} \zeta \frac{\partial}{\partial \zeta} \\
\tilde{X}_{\varphi_{-}}^{R} & =-\frac{i}{2} e^{2 i \varphi_{0}} \varphi_{+} \frac{\partial}{\partial \varphi_{0}}+e^{2 i \varphi_{0}}\left(1+\varphi_{+} \varphi_{-}\right) \frac{\partial}{\partial \varphi_{-}}+\lambda e^{2 i \varphi_{0}} \varphi_{+} \zeta \frac{\partial}{\partial \zeta}
\end{aligned}
$$

where $(A \times B)_{a} \equiv \epsilon^{a b c} A_{b} B_{c}, \epsilon^{123}=1$, denotes the vector product and $(A \times B)_{ \pm} \equiv$ $(A \times B)_{1} \pm i(A \times B)_{2}$. The commutators (13) now adopt the following form:

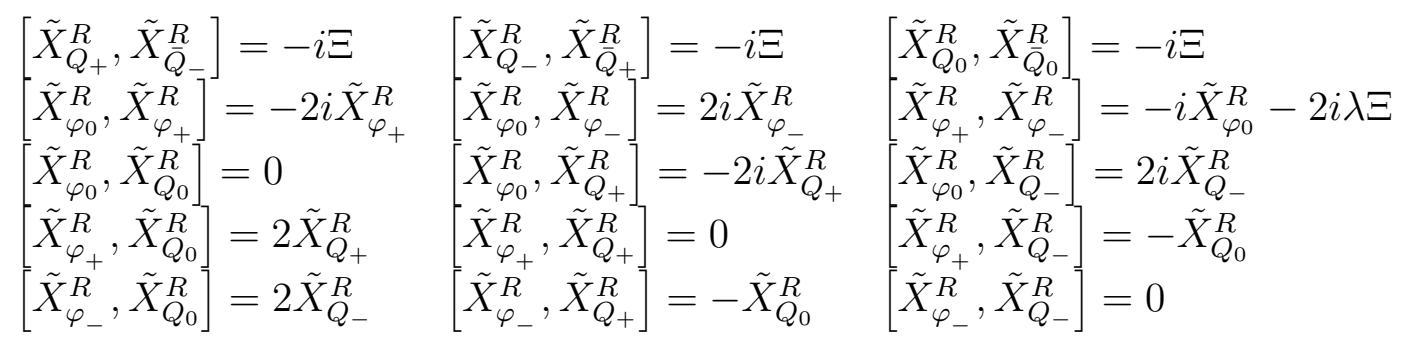

where we have omitted the commutators $\left[\tilde{X}_{\varphi_{0}, \varphi_{+}, \varphi_{-}}^{R}, \tilde{X}_{\bar{Q}_{j}}^{R}\right]$, which have the same form as for the $\tilde{X}_{Q_{j}}^{R}$ vector fields. One can also work out easily the Quantization 1-form (11), which is:

$$
\Theta=\frac{i}{4} \operatorname{tr}[\bar{D} d D-D d \bar{D}]+\overbrace{\frac{i \lambda}{1+\chi_{+} \chi_{-}}\left(\chi_{-} d \chi_{+}-\chi_{+} d \chi_{-}\right)}^{\Theta_{S U(2)}}-i \zeta^{-1} d \zeta,
$$

where we denote $D \equiv U Q U^{-1}, \bar{D} \equiv U \bar{Q} U^{-1}, \chi_{+} \equiv e^{2 i \varphi_{0}} \varphi_{+}, \chi_{-} \equiv e^{-2 i \varphi_{0}} \varphi_{-}$. The characteristic subalgebra is just

$$
\mathcal{G}_{c}=<\tilde{X}_{\varphi_{0}}^{L}>
$$

and a full-polarization subalgebra exists for arbitrary (non-zero) $\lambda$, which is:

$$
\mathcal{G}_{p}=<\tilde{X}_{\varphi_{0}}^{L}, \tilde{X}_{\varphi_{+}}^{L}, \tilde{X}_{Q}^{L}>
$$


The general solution to the polarization equations $\tilde{X}^{L} \Psi=0, \tilde{X}^{L} \in \mathcal{G}_{p}$ leads to a Hilbert space $\mathcal{H}^{(\lambda)}(\tilde{G})$ of wave functions of the form:

$$
\Psi^{(\lambda)}\left(\zeta, \varphi_{0}, \varphi_{+}, \varphi_{-}, Q, \bar{Q}\right)=\zeta\left(1+\varphi_{+} \varphi_{-}\right)^{-\lambda} e^{-\frac{1}{4} \operatorname{tr}[\bar{Q} Q]} \Phi\left(\chi_{-}, \bar{D}\right)
$$

where $\Phi$ is an arbitrary power series in the variables $\chi_{-}$and $\bar{D}$. A scalar product can be given through the invariant integration volume of $\tilde{G}$ :

$$
\mu(\tilde{g})=\frac{-i}{\left(1+\varphi_{+} \varphi_{-}\right)^{2}}\left[\prod_{a=1}^{3} d A_{a} \wedge d E_{a}\right] \wedge\left[d \operatorname{Re}\left(\varphi_{+}\right) \wedge d \operatorname{Im}\left(\varphi_{+}\right) \wedge d \varphi_{0}\right] \wedge \zeta^{-1} d \zeta .
$$

The phase space related to this quantum system is clearly $\Re^{3} \times \Re^{3} \times S^{2}$, as can be inferred from the symplectic form $\omega \equiv d \Theta / \mathcal{G}_{c}$ (the quotient of $d \Theta$ by the trajectories generated by left-invariant vector fields in (41)), the parameter $\lambda$ being the analogous of the spin $s$.

The constraint equations

$$
\begin{aligned}
& \tilde{X}_{\varphi_{0}}^{R} \Psi_{\text {phys. }}^{(\lambda)}=0 \Rightarrow \chi_{-} \frac{\partial \Phi}{\partial \chi_{-}}+i\left(\bar{D} \times \frac{\partial \Phi}{\partial \bar{D}}\right)_{0}=0 \\
& \tilde{X}_{\varphi_{-}}^{R} \Psi_{\text {phys. }}^{(\lambda)}=0 \Rightarrow \frac{\partial \Phi}{\partial \chi_{-}}+i\left(\bar{D} \times \frac{\partial \Phi}{\partial \bar{D}}\right)_{+}=0
\end{aligned}
$$

keep 2 degrees of freedom out of the original $4=3+1$ corresponding to this "spinninglike particle". They can be interpreted as zero total angular-momentum (orbital+spin) conditions. Note that the condition

$$
\tilde{X}_{\varphi_{+}}^{R} \Psi_{\text {phys. }}^{(\lambda)}=0 \Rightarrow-2 \lambda \chi_{-} \Phi+\chi_{-}^{2} \frac{\partial \Phi}{\partial \chi_{-}}-i\left(\bar{D} \times \frac{\partial \Phi}{\partial \bar{D}}\right)_{-}=0
$$

is incompatible with both conditions in (45), which correspond to a polarization subalgebra $\mathcal{T}_{p}=<\tilde{X}_{\varphi_{0}}^{R}, \tilde{X}_{\varphi_{-}}^{R}>$ of $\mathcal{T}$, unless $\lambda=0$. For $\lambda=0$, the characteristic subalgebra (41) contains the whole $s u(2)$ subalgebra, $\Phi$ does no longer depend on $\chi_{-}$, and the constraint conditions $(45,46)$ keep a "radial" dependence of $\Phi$ on $R^{2} \equiv \frac{1}{2} \operatorname{tr}[D \bar{D}]$ ("s-waves"), as corresponds to a spin-zero particle with zero orbital angular momentum.

The good operators are

$$
\tilde{\mathcal{G}}_{\text {good }}=<\operatorname{tr}\left[\hat{Q}^{2}\right], \operatorname{tr}\left[\hat{\bar{Q}}^{2}\right], \operatorname{tr}[\hat{Q} \hat{\bar{Q}}], \hat{C}, \Xi>
$$

where $\hat{C}=\left(\tilde{X}_{\varphi_{0}}^{R}+2 \lambda \Xi\right)^{2}+2 \tilde{X}_{\varphi_{+}}^{R} \tilde{X}_{\varphi_{-}}^{R}+2 \tilde{X}_{\varphi_{-}}^{R} \tilde{X}_{\varphi_{+}}^{R}$ is the Casimir operator of $S U(2)$.

\section{Acknowledgment}

M. Calixto would like to thank the University of Granada for a Post-doctoral grant and the Department of Physics of Swansea for its hospitality. 
Note added. We thank the referee who brought the reference [16] to our attention. It contains a nice summary of a generalization of Dirac's method of quantization of constrained systems by using Mackey's theory of inequivalent quantizations on a coset space $G / H$. The reader may find it interesting to compare GAQ and this generalized version of Dirac's approach by using the simple example given in the Appendix. Both approaches share the idea of "emergence of new (internal) degrees of freedom, existence of inequivalent quantizations and the appearance of an $H$-connection" when constraints become second class. In fact, the role played by the characteristic subgroup $G_{c}$ in GAQ is similar to the role played by $H$ when quantizing on a coset space $G / H$; also, the piece $\Theta_{S U(2)}=\left.\frac{\partial}{\partial g^{j}} \xi\left(g^{\prime} \mid g\right)_{\lambda}\right|_{g^{\prime}=g^{-1}} d g^{j}$ of the general connection form (11) in Eq. (40) corresponds to a "SU(2)-connection". However, an important distinction has to be made between both schemes of constrained quantization. The counterpart of the constraint equations (right conditions)

$$
R_{h} \Psi(g)=\Psi(g * h) \equiv \Psi(g), \forall h \in H, g \in G,
$$

in the generalized Dirac's approach to the constrained quantization on $G / H$, are the polarization equations of GAQ (see paragraph before Eq. (14)) which, in contrast, are intended to reduce the (left) regular representation $L_{g^{\prime}} \Psi(g)=\Psi\left(g^{\prime} * g\right)$ of $G$ on wave functions $\Psi$. In brief, GAQ further "constrains" wave functions by means of extra $T$ equivariance conditions (8) like (45), which are not present in the generalized Dirac's scheme of quantization. Also, T-equivariance conditions in GAQ force the definition of good operators (observables), concept which is absent in the other scheme.

\section{References}

[1] V. Aldaya and J. de Azcárraga, J. Math. Phys. 23, 1297 (1982).

[2] M. Navarro, V. Aldaya and M. Calixto, J. Math. Phys. 38, 1454 (1997); J. Math. Phys. 37, 206,(1996).

[3] Carlo Rovelli, Phys. Rev. Lett. 80, 4613 (1998).

[4] V. Aldaya, M. Calixto and M. Navarro, Int. J. Mod. Phys. A12, 3609 (1997).

[5] M. Calixto and V. Aldaya, Gauge transformation properties of vector and tensor potentials revisited: a group quantization approach, preprint hep-th/9903106.

[6] V. Aldaya, J. Navarro-Salas and A. Ramírez, Commun. Math. Phys. 121, 541 (1989).

[7] R. Jackiw and C. Rebbi, Phys. Rev. Lett. 37, 172 (1976), C. Callan, R. Dashen and D. Gross, Phys. Lett. B63, 334 (1976).

[8] R. Jackiw, Topological investigations of quantized gauge theories, in "Current Algebra and Anomalies", eds. S.B. Treiman et al. (World Scientific, 1985). Rev. Mod. Phys. 52, $661(1980)$. 
[9] V. Aldaya, J. Guerrero, G. Marmo, Quantization of a Lie Group: Higher Order Polarizations, in "Symmetries in Science X", Ed. Bruno Gruber and Michael Ramek, Plenum Press New York (1998); physics/9710002.

[10] V. Aldaya and J. Navarro-Salas, Commun. Math. Phys. 139, 433 (1991).

[11] M. Bauer, D. Z. Freedman and P. E. Haagensen, Nucl. Phys. B428, 147 (1994);

D. Z. Freedman, Nucl. Phys. (Proc. Suppl.) 39B,C, 447 (1995).

[12] Carlo Rovelli, Phys. Rev. D42, 2638 (1990).

[13] V.G. Kac, Contravariant form infinite dimensional Lie algebras and superalgebras, Lecture Notes in Physics, 94, 441 (1979).

[14] B.L. Feigin and D.B. Fuchs, Funct. Anal. Appl. 16, 114 (1982).

[15] N.P. Landsman and N. Linden, Nucl. Phys. B365, 121 (1991).

[16] D. McMullan and I. Tsutsui, Ann. Phys. 237, 269 (1995).

[17] V. Aldaya, M. Calixto and J. Guerrero, Commun. Math. Phys. 178, 399 (1996).

[18] V. Aldaya, M. Calixto and J.M. Cerveró, Commun. Math. Phys. 200, 325 (1999).

[19] V. Aldaya and J. de Azcárraga, J. Phys. A16, 2633 (1983).

[20] E.G. Floratos, J. Iliopoulos and G. Tiktopoulos, Phys. Lett. B217, 285 (1989). 